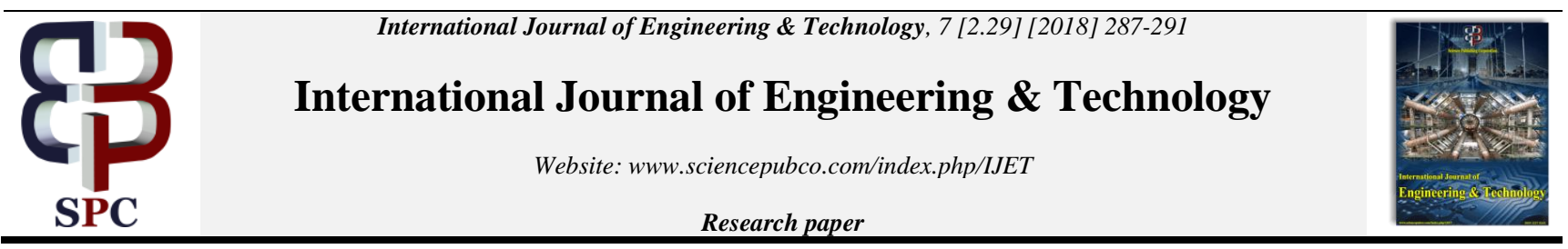

\title{
The association of maternal diet and polyamines in human milk: a study among malay ethnic mothers in kuantan, malaysia
}

\author{
Puteri Amirah Adib kamaruzzaman', Radiah Abdul Ghani ${ }^{2,}$, Muhammad Ibrahim³, Nor Azwani Mohd. Shukri ${ }^{4}$ \\ ${ }^{1,2}$ Department of Biomedical Science, Kulliyyah of Allied Health Sciences, International Islamic University Malaysia, \\ Kuantan Pahang Malaysia \\ 3,4, Department of Nutrition Science, Kulliyyah of Allied Health Sciences, International Islamic University Malaysia, \\ Kuantan Pahang Malaysia \\ *Corresponding author E-mail: radiah@iium.edu.my
}

\begin{abstract}
Human milk contains a lot of nutrient and it offers advantages to infant age less than six months. Polyamines in milk accelerate the infants' gut maturation and protect babies from the harsh environment. Maternal diet is a factor which can affect the polyamines variability in milk. To date, no research on local diet and the composition of human milk has been investigated. This study aimed to identify an association between Malaysian maternal diet and composition of polyamines in human milk. Seventy nursing mothers were recruited in Pahang and each mother recorded their 24-hours food consumption within a week. Maternal diets were recorded in Nutritionist Prosoftware and the macronutrients were calculated. Milk samples were collected from each mother after $24 \mathrm{~h}$ of food diary. Polyamines in milk were extracted using $0.4 \mathrm{M}$ Perchloric acid, dansylated and quantified using HPLC. The mean age of the respondent was 30.13 years $[\mathrm{SD}=3.145]$.Nursing mothers consumed high carbohydrate [41.4\%] compared to fat [23.1\%] and protein [13.7\%].Spermidine [49.1\%] was the highest polyamines in the breast milk samples. A significant correlation were shown between putrescine and dietary carbohydrate $[\mathrm{p}=0.027]$ and putrescine and dietary protein $[\mathrm{p}=0.031]$.The maternal education levels has no association with maternal diet pattern and breastfeeding practice in this study [p=0.657]. It is suggested that polyamines composition in human milk may be modulated by carbohydrate and protein intake among maternal mother
\end{abstract}

Keywords: Polyamines; maternal diet; Malaysia; human milk

\section{Introduction}

The World Health Organization [1] and United Nations Children's Fund [2] strongly advocate exclusive breastfeeding for the first six months after birth as the optimal way of feeding infants. This is based on evidence which demonstrated its benefits on child health, nutritional, immunologic, developmental, psychological, social, economic, and environmental status, as well as, its positive implications on maternal wellbeing [3]. The Malaysian government has adopted this recommendation in its Malaysian Dietary Guidelines [MDG] which encourage mothers to practice breastfeeding from birth until six months and continue to breastfeed for two years [4, 5]. The recommendation has been emphasized as Key Message 1 in the recent MDG 2013. Despite the well-known advantages of breastfeeding, percentage of infants' age less than six months being exclusively breastfeeding in Malaysia is still low at $14.5 \%$, which were last reported in NHMS III 20061[5]. There are a number of reasons related to the inability to continue with breastfeeding among some mothers. The most common cause of early termination of breastfeeding is the perception of insufficient milk production by the mother or insufficient milk intake by the baby [6]. In addition, lack of information on specific composition and its crucial function has been reported to influence breastfeeding cessation [7].

Concerning this issue, there are investigations have been conducted to determine the composition of human milk. One of the cofactors that are highly important is the polyamines. Polyamines, which comprises putrescine, spermidine and spermine has been found to be higher in concentration compared to cow, camel and goat's milk [8]. Polyamines are a critical nutrient which promotes the maturation of infant's intestine during the six months of infant's life. Immature intestine can leads to many problems such as incomplete digestion, inflammatory response and diarrhea in infants. It has been found that breastfed premature infants have lower intestinal permeability compared to infants who did not breastfeed. Reduction of the intestinal permeability of macromolecules was found to be able to reduce occurrence of allergic reactions of food in children [9].

The level of polyamines in human milk is affected by several factors. Scientists have found that these variabilities may be due to individual differences such as age of the mother, genetic influence, external environment factor and maternal diet. Since maternal diet is the easiest way to be manipulated and Malaysia has unique distinct eating patterns from any other countries, this factor has been chosen for this study. Moreover, no such study has been conducted in Malaysia. Therefore, this study aimed to determine if there is any association between maternal diet which includes macronutrients [protein, carbohydrate and lipid] and the polyamines [putrescine, spermidine and spermine] levels in human milk among nursing mothers in selected area in Pahang. Besides, the association between maternal educational level with maternal diet and breastfeeding practice were also being identified. 


\section{Literature Review}

\subsection{Recommendation of breastfeeding}

Breastfeeding is very important to prepare the infant from intrauterine to extrauterine life. Infant will take at least one year for his gastrointestinal tract to significantly develop and mature [10]. Due to this, World Health Organization [1] recommended mothers to exclusively breastfeed their infants for at least six months. Exclusive breastfeeding means the infant depends solely on mother's milk without other additional food or drink, including water [4]. Breast milk is more than enough for the infant to obtain energy and nutrients needed. In Malaysia, Malaysia National Breastfeeding policy also encouraged Malaysian mothers to breastfeed their child with breast milk exclusively especially for infants less than six month or until their child reach two years old [11]. Besides, the Ministry of Health $[\mathrm{MOH}]$ in Malaysia has taken a lot of proactive steps in promoting breastfeeding practice, especially through the implementation of Baby Friendly Hospital Initiative [BFHI]. BFHI is a global initiative by WHO and UNICEF and has been carried out in Malaysia since 1993[12]. The Malaysian government has adopted this recommendation in its Malaysian Dietary Guidelines [MDG] which encourage mothers to practice breastfeeding from birth until six months and continue to breastfeed for two years [2-3]. The recommendation has been emphasized as Key Message 1 in the recent MDG 2013.Malaysia also gained supports from non-government organization [NGO] such as The Malaysian Breastfeeding Peer Counselor [MBfPC], who offers support groups services, meet-ups, seminars and advice service to breastfeeding mothers.

\subsection{Composition of human milk}

Human milk is a complex fluid containing various components needed by an infant for the first six months of life. Components of human milk can be divided into nutritional component, bioactive component, growth factor and immunological factors [13]. This study focused more on the nutritional components of human milk and they are classified to micronutrient and macronutrient. Micronutrient such as Vitamin A, B1, B2, B6, B12, D and iodine are needed by the body in micro amount. On the other hand, macronutrient such as carbohydrates, proteins, and fats are nutrients that are needed in large amount for energy. [13] estimated that the general human milk mean composition of mother with full term baby for protein is 0.9 to $1.2 \mathrm{~g} / \mathrm{dL}, 3.2$ to $3.6 \mathrm{~g} / \mathrm{dL}$ for fat, and lastly lactose with 6.7 to $7.8 \mathrm{~g} / \mathrm{dL}$. Interestingly, human milk composition can modify itself according to the needs of the baby during growth and maturation [14]. For example, during the first two and three days after delivery, special milk called colostrum is secreted. Colostrum contains antibodies, high concentration of protein, minerals and fat-soluble vitamins to increase the baby's immunity. After colostrum phase is over, transitional milk will be produced in a big amount to serve the baby's growth needs. Transitional milk contains higher levels of fat, lactose and calories. Lastly, the mature milk contains more water than carbohydrates and proteins which are important for the baby's development and energy.

\subsection{Factors contribute toward quality and quantity of human milk}

[13] believed components of the human milk vary depending on maternal diet and body stores. Despite variation in the maternal diet, macronutrient composition across populations is remarkably maintained. Composition of human milk may also differ according to the several influences such as maternal body weight and height, protein intake, parity, menstruation and breastfeeding rates $[1,13]$. For example, mother with preterm baby is found to produce significantly higher content of protein in their milk when compared to mother will full term baby $[13,15]$. Meanwhile, [16] reported that mother with preterm baby has a significantly lower lactose concentration compared to mother with full term baby.

In addition, a carbohydrate called oligosaccharides was correlated to vary among mothers due to the genetic basis of the mother's Lewis blood group[16] [16]. In the report,[16] also indicated that the composition of lipid in human milk has a greater variability compared to other macronutrient. He claimed that the composition of lipid in human milk may be determined by the mother's body mass and parity. Parallel to [17], [16] also found that in another study conducted in Malaysia, the researcher found a significant different between human milk fatty acid profile with the mother's ethnicity, which may be due to the different maternal diet between the ethnics. As yet, little study has been done to correlate the association of maternal diet in Malaysia with composition of the human milk.

\subsection{Maternal Diet and Human Milk}

Maternal diet means the customary amount and of food and drink taken by a mother to meet specific requirements during lactation [18]. Meanwhile, lactation means the secretion of milk [18]. [19] defined lactation as the life phase of an infant in which human milk is the provider for the infant's growth needs. Breastfeeding and recovering mother requires a lot of energy as she is feeding herself and her infant. According to [2] breastfeeding mother needs extra 500 calories per day compared to her pregnancy day. Food and Agriculture of the United Nations [2001] revealed that the prerequisite energy of lactating mother is equal to the energy intake from the maternal diet. Generally, maternal diet does not affect how much milk will be produced per day [2, 20]. Similarly for undernourished mother, existing data since 1977 proposed that undernourished mothers will not affect the duration of breastfeeding and the mother can produce enough milk for their infants.

However, the only difference that can be affected by maternal diet is the quality of the milk produced. In study done by [21], it is found that mothers with low fat, high carbohydrate diet was found to have higher concentration of milk with medium chain fatty acid. This study suggested the low fat and high fat diet has significant impact on fatty acid composition in human milk. Another study done by [22], also revealed the same conclusion when they found fatty acid profile in European mother's milk influenced by certain dietary factor. In another study by [23], they reported that dairy cows show a significant relationship between carbohydrate restriction and reduced milk production.

\subsection{Polyamines in milk}

During the neonatal period, newborn's intestines are still immature. Being unprotected and exposed to various type of microorganisms and antigens, maturation of the infants' gut is very crucial to survive the harsh environment. Moreover, the maturation of the infant's intestine is important to prepare the drastic change in baby diets from milk to solid food. In order to overcome the problems, breastfeeding plays a significant role as mother's milk is the gold exogenous source of polyamines for the neonates to obtain nutrient and protection for the baby. [8] concluded that polyamines will speed up the neonates' intestinal maturation and proliferation process. [24] animal experiment revealed that polyamines in breast milk enhanced the maturation of experimental suckling rat's intestinal and systemic immune system by facilitating the maturation process of intraepithelial lymphocytes and lamina propria lymphocytes construction. Complete intestinal maturation will result in increased mucus and digestive enzyme production, immunological adaptation, decreased gut permeability and increase intraepithelial lymphocytes.

Dandrifosse et al. [25] [as cited in [9] found that breastfed premature infants showed to have lower intestinal permeability compared to infants who do not breastfeed. Reduction of the intestinal permeability of macromolecules were found to be able to reduce occurrence of allergic reactions of food in children $[13,26]$. 
There are a lot of studies had been done and show considerable individual variation of polyamines concentration in milk. Research by $[15,27,28]$ revealed that variation in the human milk was due to individual variabilities such as age of the mother, genetic influence, ethnic origin, circadian rhythm of polyamines production, nutritional status, amount of dietary polyamines intake, duration of lactation, environment influences, amount of milk in the breast and many more. They also claimed maternal body weight for height, protein intake, parity, return of menstruation and nursing frequency also contributed to the variation in macronutrient concentration in human milk.

Furthermore, synthesis of polyamines in the mother's mammary gland also can increase gradually during gestation and lactation [27]. In recent years, several studies have been done by the western countries to evaluate the maternal diet on milk content production, but only limited studies had been done so far on polyamines. Particularly for this study, a strong correlation was found between the maternal diet and polyamines content in human milk of mother with preterm baby [27, 28].In animal study, an increase of rat milk polyamines levels were found in rats fed orally with additional spermidine, spermine and amino acid [27]. This study is in accordance with $[27,28]$.

For the first time, this study aimed to investigate the association of local maternal diet with polyamines among nursing mothers in Pahang. It is hope that this outcome contributes additional scientific knowledge in order to highlight another benefit of breastfeeding in Malaysia.

\section{Methodology}

\subsection{Ethical Approval}

Ethical approval was obtained from the University Kulliyyah of Allied Health Sciences Research Committee and IIUM Research Ethical Committee [IREC] before this cross sectional study was conducted.

\subsection{Setting and Participants}

This study was conducted in Kuantan, Malaysia between December 2015 and December 2016. It has been conducted among mothers who practiced exclusive breastfeeding from age 18 to 40 years old. Power and Sample Size Calculations [PS] software was used to calculate the sample size in this study. The calculation using independent $t$ - test, $\alpha$ value is 0.05 , power $=0.8, \delta=3.7, \sigma=5.32$ and $\mathrm{m}=1$ that yields 70 subjects. Therefore, 70 mothers were selected purposively from various healthcare settings in Kuantan Pahang.

\subsection{Inclusion Criteria}

These include nursing mothers aged between 18 to 40 years old, or women of the mentioned age, who are exclusively breastfeeding a baby aged six months or younger.

\subsection{Exclusion Criteria}

Mothers who have history of chronic diseases such as hypertension, diabetes mellitus or cardiovascular disease were excluded from the study

\subsection{Macronutrient Intake Analysis}

Mothers' 24 hours food and beverage consumptions were recorded in Food Diary [FD] for three days [two weekdays and one weekend]. One ounce of milk sample was self-expressed by the mother at every next day of FD was recorded. Macronutrients of maternal diet recorded were calculated automatically using Nutritionist Pro. Software

\subsection{Polyamine Extraction from Milk Samples}

Firstly, the fat component of the human milk was removed using centrifuge. Polyamines in the human milks were then extracted by adding ice cold PCA solution into the human milk sample in a ratio of $1 \mathrm{ml}$ of sample to $0.6 \mathrm{ml}$ of PCA. The mixture was vortex thoroughly and incubated in $4^{\circ} \mathrm{C}$ fridge for 15 minutes before it was centrifuged again at $13000 \mathrm{~g}$ for 15 minutes at $4^{\circ} \mathrm{C}$. Clear upper supernatant produced was transferred into new tube for dansylation. Extracted samples were spiked with $0.001 \mathrm{ml}$ internal standard prior dansylation process continued. The extracted polyamines were then subjected to a derivatization by adding $0.05 \mathrm{ml}$ of NCDH and $0.5 \mathrm{ml}$ of dansyl chloride. The mixture was incubated overnight in a dark water bath at $25^{\circ} \mathrm{C}-37^{\circ} \mathrm{C}$. Excess dansyl chloride was removed by adding $0.125 \mathrm{ml}$ of proline to all samples and incubated in $37^{\circ} \mathrm{C}$ water bath until the solutions turns pale yellow or colourless. Subsequently, $0.5 \mathrm{ml}$ of toluene was added to extract dansylated polyamines and left under fume hood to evaporate. Finally, $0.07 \mathrm{ml}$ of reconstitute sample was transferred into a HPLC vial and ready to be assayed immediately.

\subsection{Polyamine Quantification by using HPLC}

Twenty $\mu$ of dansylated samples from each vial was injected into HPLC with stationary phase of column C18 and mobile phase of $40 \%[\mathrm{v} / \mathrm{v}]$ acetonitrile and $60 \%[\mathrm{v} / \mathrm{v}]$ water. Mobile phase flow at $1.0 \mathrm{ml} / \mathrm{min}$. Wavelengths of excitation and emission in fluorescence spectrophotometer [Agilent Technologies] were set at 347 $\mathrm{nm}$ and $465 \mathrm{~nm}$ respectively.

\subsection{Statistical Analysis}

All data in the questionnaire were coded and entered into the Statistical Package for the Social Sciences [SPSS] and statistical calculation were performed using SPSS. Statistical test used in this study was one sample t-test, Kruskal-Wallis test, Fisher's Exact test and Pearson's correlation test. A p-value of less than 0.05 was considered significant. Parametric data were presented as mean \pm standard deviation [SD] while non-parametric data were presented in median [interquartile range] [IQR].

\section{Results And Findings}

\subsection{Demographic Data Analysis}

Maternal participants' ages were averages of $30.13 \pm 4.5$ with all are Malay ethnicity. Out of this number, $66 \%$ of them had at least a degree, while others completed their primary or secondary school education. The mothers household income where $19 \%$ of them with household income of low category, another $67 \%$ with household income in a middle and $14 \%$ of them with household income more than RM 5000.

\subsection{Macronutrient Intake Analysis}

This present study found that the nursing mothers in Pahang ate a high carbohydrate diet. Based on Figure 1, nursing mothers consumed carbohydrate the most with mean of approximately 259.06 $\mathrm{g}$ per day followed by protein intakes of $85.70 \mathrm{~g}$ per day. Fat intakes of the nursing mothers were at the least in which they only consumed $64.18 \mathrm{~g}$ fat per day. Nursing mothers' energy and carbohydrate intakes were significantly lower than the recommended nutrient intake [RNI]. On the other hand, protein intakes have exceeded the recommended level while fat intakes were in recommended range.

Table 1: Socio-demographic characteristic of nursing mothers

\begin{tabular}{|l|c|c|}
\hline \multirow{2}{*}{ Characteristics } & \multicolumn{2}{|c|}{ Number of respondents } \\
\cline { 2 - 3 } & No. & Percentage [\%] \\
\hline Race & & \\
\hline Malay & 70 & 100 \\
\hline
\end{tabular}




\begin{tabular}{|l|c|c|}
\hline Education & & \\
\hline Low [Primary and secondary school] & 24 & 34 \\
\hline High [Degree, master and PhD holder] & 46 & 66 \\
\hline Household income & & \\
\hline < RM 1000 & 13 & 19 \\
\hline RM 1000 - RM 3000 & 24 & 34 \\
\hline RM 3000 - RM 5000 & 23 & 33 \\
\hline$>$ RM 5000 & 10 & 14 \\
\hline
\end{tabular}

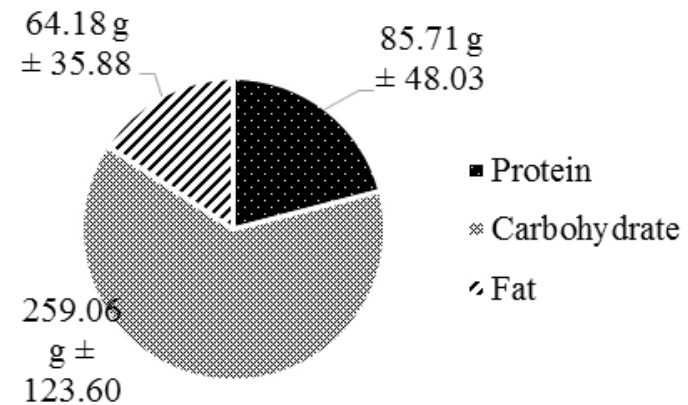

Fig. 1: Macronutrients mean intake by the seventy nursing mothers in Kuantan, Pahang

However, it was apparent from Figure 2 that the nursing mothers' energy and carbohydrate intakes were significantly lower than the RNI by MOH although carbohydrate contributes the most in energy composition. The author thought that this low energy intake was due to the fact that the nursing mothers only perform light physical activity and only small amount of energy was needed by the nursing mothers in developing country.

\subsection{Analysis on polyamines in human milk}

This study focused specifically on polyamines which is crucial in cell proliferation and differentiation especially in promoting infant's organ development. Figure 3 demonstrates spermine as the lowest concentration of total polyamines which is at $11.23 \%$. Putrescine is $28.44 \%$ higher than spermine. Spermidine has the highest concentration level compared to the other two which comprises $49.1 \%$ of total polyamines. A study by [27]reported that in an in vivo animal study, more than $90 \%$ of exogenous spermidine was discovered in breast milk of the mouse in a period of 24-48 hours while $70 \%$ of putrescine was assumed to transform to spermidine. This might explain the high level of spermidine in the human milk compared to other polyamines.

\subsection{Correlation between polyamines and macronutri- ents intake}

Polyamines particularly putrescine were reported to run parallel with level of protein in the human milk [27]. As seen in Table 2, fair correlation was found between the level of putrescine and protein [p-value $=0.057$ ]. While for spermine and spermidine, the correlation was not observed. This present study is in agreement with their claim when most of spermine was below detection level, indicating their small quantity in the human milk. These results proposed that compared to other polyamines, spermine may have its own unique metabolism and importance.

Table 2 also revealed that there is positive correlation between carbohydrate and putrescine $[\mathrm{p}=0.027]$. This finding suggests that as the intake of dietary carbohydrate is increase, level of putrescine in the breast milk will also increase. Previous claim indicates that fruits and cereals, which are the well-known major source of carbohydrate, may have a positive relationship with the production of putrescine in the breast milk.
Table 2: Pearson's Correlation [R] of Macronutrients with Polyamines

\begin{tabular}{|c|c|c|c|}
\hline \multirow{2}{*}{ Polyamines } & \multicolumn{3}{|c|}{ R-value $[\boldsymbol{p}$-value $]$} \\
\cline { 2 - 4 } & Protein & Carbohydrate & Fat \\
\hline Putrescine & $0.378[0.057]^{*}$ & $0.434[0.027]^{*}$ & $0.171[0.404]$ \\
\hline Spermidine & $-0.088[0.720]$ & $-0.064[0.796]$ & $-0.056[0.820]$ \\
\hline Spermine & $0.157[0.766]$ & $0.240[0.646]$ & $0.045[0.933]$ \\
\hline
\end{tabular}

\subsection{The relationship between educational background and macronutrient intake}

Table 3 shows association of macronutrients intake by the nursing mothers with their level of education using SPSS Kruskal-Wallis test. None has shown any significant association between macronutrients intake and the nursing mothers' education level. A Fisher Exact's tests were also conducted as shown in Table 4, however no significant association between level of education and practice of breastfeeding found in this study.

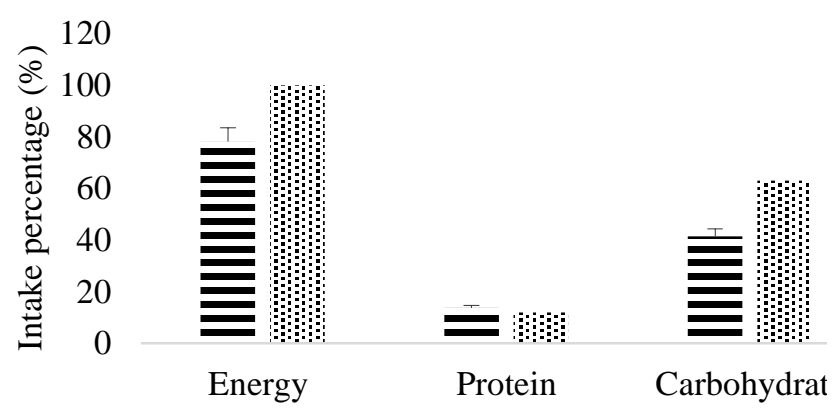

Fig. 2: Comparison of macronutrients intake by nursing mothers with RNI by the $\mathrm{MOH}$

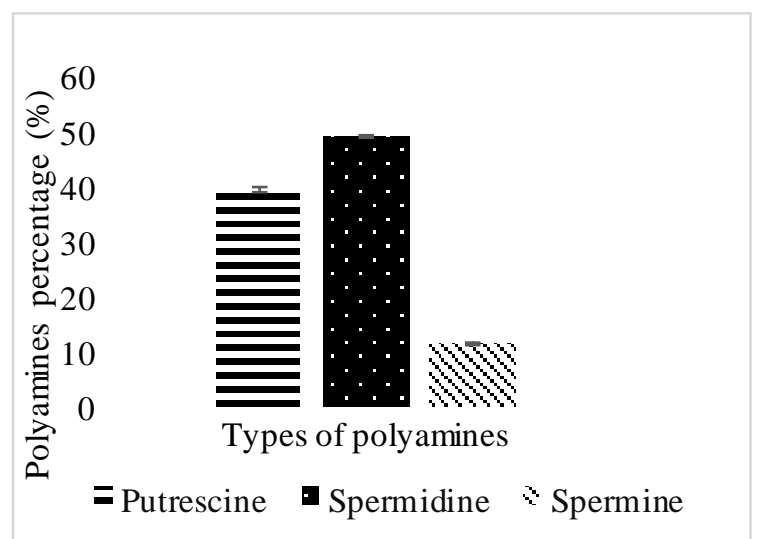

Fig. 3: Composition of polyamines in the seventy nursing mothers' breast milk

There are many factors that can affect the pattern of maternal diet including cultural norm, demographic status, socioeconomic level and many more. In this study, the association of mother's education level with the maternal diet was determined. According to Table 3, none has shown any significant association between macronutrients intake and the nursing mothers' education level. A similar investigation has been conducted in United States which claimed people with higher education level has a better health and better access to healthy food. food [28] also supported this claim when they reported people with lower socioeconomic groups have lower awareness in getting healthier food. Contrary to this expectation, this present study nonetheless found no significant association between the mothers' education level with their macronutrients intake. This finding suggests that it may be due to the random selection of the subject among urban educated nursing mothers. Hence the variability of maternal diet is restricted. 
Table 3: Comparison Table of Energy, Protein, Carbohydrate and Fat Intake with Education Levels of the Nursing Mothers

\begin{tabular}{|c|c|c|c|}
\hline Macronutrient & Median [IQR] & $\begin{array}{c}\text { Kruskal-Wallis test } \\
{[\mathbf{d f}]}\end{array}$ & $\begin{array}{c}\boldsymbol{p} \text { - } \\
\text { value }\end{array}$ \\
\hline Energy & $\begin{array}{c}1740.74 \\
{[1030.41]}\end{array}$ & $1.664[3]$ & 0.645 \\
\hline Protein & $65.87[54.11]$ & $0.834[3]$ & 0.841 \\
\hline Carbohydrate & $229.23[160.64]$ & $2.024[3]$ & 0.567 \\
\hline Fat & $49.53[50.34]$ & $1.151[3]$ & 0.765 \\
\hline
\end{tabular}

\subsection{Limitations of Study}

This study was a preliminary analysis with purposive sampling, conducted in a few healthcare settings and clinics in Kuantan Pahang Malaysia. Thus, the result may not be giving the reflection of the general population.

\section{Conclusion}

Improving scientific information of the benefits of human milk can improve the attitudes of mothers towards breastfeeding practice especially for infants below than six months. This study revealed the local diet among selected population in Kuantan, Malaysia correlated with high spermidine level in human milk. More importantly, a fair correlation was found between putrescine and carbohydrate, suggesting that the intake of dietary carbohydrate can influence the content of putrescine in the human milk. This study adds to the body of knowledge regarding maternal diet and its association with the polyamines content in human milk. Future research should additionally investigate the polyamines in human milk based on control parameter which compare nursing mothers with poor diet and nursing mothers with rich heathy diet and controlled study between ethnicity It is hope that these findings leads to better understanding in this issue and able to provide data for policy makers.

\section{Acknowledgement}

This work was supported by the MIRGS 13-01-001-0004 grant from Ministry of Higher Education. The authors wish to acknowledge Kulliyyah of Allied Health Sciences and Kulliyyah of Pharmacy, IIUM for providing facilities for this project.

\section{References}

[1] WHO. World Health Organization. [2014]. Comprehensive Implementation Plan on Maternal, Infant and Young Child Nutrition. . In: Organization WH, editor. USA2014.

[2] UNICEF. Related maternal nutrition. In: Fund UNICsE, editor. 2015.

[3] Kramer MS, Kakuma R. Optimal duration of exclusive breastfeeding. Cochrane Database Syst Rev. 2012;8[8]:CD003517.

[4] McGuire S. World Health Organization. Comprehensive Implementation Plan on Maternal, Infant, and Young Child Nutrition. Geneva, Switzerland, 2014. Advances in Nutrition: An International Review Journal. 2015;6[1]:134-5.

[5] NCCFN. Recommended Nutrient Intakes for Malaysia. A Report of the Technical Working Gropu on Nutritional Guidelines. 2005.

[6] Wang W, Lau Y, Chow A, Chan KS. Breast-feeding intention, initiation and duration among Hong Kong Chinese women: a prospective longitudinal study. Midwifery. 2014;30[6]:678-87.

[7] Oakley LL, Henderson J, Redshaw M, Quigley MA. The role of support and other factors in early breastfeeding cessation: an analysis of data from a maternity survey in England. BMC pregnancy and childbirth. 2014;14[1]:88.

[8] Ali MA, Strandvik B, Palme-Kilander C, Yngve A. Lower polyamine levels in breast milk of obese mothers compared to mothers with normal body weight. Journal of Human Nutrition and Dietetics. 2013;26[s1]:164-70.

[9] Larqué E, Sabater-Molina M, Zamora S. Biological significance of dietary polyamines. Nutrition. 2007;23[1]:87-95.
[10] Anderson RC, Dalziel JE, Gopal PK, Bassett S, Ellis A, Roy NC. The role of intestinal barrier function in early life in the development of colitis. Colitis: InTech; 2012.

[11] MOH. Inisiatif Hospital Rakan Bayi. 2015a.

[12] MOH. Malaysian Dietary Guidelines for Children and Adolescents. National Coordi nating Committee on Food and Nutrition. 2013.

[13] Ballard O, Morrow AL. Human milk composition: nutrients and bioactive factors. Pediatric Clinics of North America. 2013;60[1]:49.

[14] Jabed A, Wagner S, McCracken J, Wells DN, Laible G. Targeted microRNA expression in dairy cattle directs production of $\beta$ lactoglobulin-free, high-casein milk. Proceedings of the National Academy of Sciences. 2012;109[42]:16811-6.

[15] Plaza-Zamora J, Sabater-Molina M, Rodriguez-Palmero M, Rivero M, Bosch V, Nadal JM, et al. Polyamines in human breast milk for preterm and term infants. British Journal of Nutrition. 2013;110[3]:524-8.

[16] Stam J, Sauer PJ, Boehm G. Can we define an infant's need from the composition of human milk? The American journal of clinical nutrition. 2013;98[2]:521S-8S.

[17] Daud AZ, Mohd-Esa N, Azlan A, Chan YM. The trans fatty acid content in human milk and its association with maternal diet among lactating mothers in Malaysia. Asia Pacific journal of clinical nutrition. 2013;22[3]:431-42.

[18] Dorland WAN. Dorland's Illustrated Medical Dictionary32: Dorland's Illustrated Medical Dictionary: Elsevier Health Sciences; 2011.

[19] Aranceta J. Spanish food patterns. Public health nutrition. 2001;4[6a]:1399.

[20] Thomson AM, Black AE. Nutritional aspects of human lactation Bulletin of the World Health Organization. 1975;52[2]:163.

[21] Nasser R, Stephen AM, Goh YK, Clandinin MT. The effect of a controlled manipulation of maternal dietary fat intake on medium and long chain fatty acids in human breast milk in Saskatoon, Canada. International breastfeeding journal. 2010;5[1]:3.

[22] Antonakou A, Skenderi KP, Chiou A, Anastasiou CA, Bakoula C, Matalas A-L. Breast milk fat concentration and fatty acid pattern during the first six months in exclusively breastfeeding Greek women. European journal of nutrition. 2013;52[3]:963-73.

[23] Heinig MJ, Doberne K. Weighing the risks: the use of lowcarbohydrate diets during lactation. Sage Publications Sage CA: Thousand Oaks, CA; 2004.

[24] Pérez-Cano FJ, González-Castro A, Castellote C, Franch À, Castell M. Influence of breast milk polyamines on suckling rat immune system maturation. Developmental \& Comparative Immunology. 2010;34[2]:210-8.

[25] Dandrifosse G, Peulen O, El Khefif N, Deloyer P, Dandrifosse AC, Grandfils C. Are milk polyamines preventive agents against food allergy? Proceedings of the Nutrition Society. 2000;59[1]:816.

[26] Löser C. Polyamines in human and animal milk. British Journal of Nutrition. 2000;84[S1]:55-8

[27] Atiya Ali M, Strandvik B, Sabel KG, Palme Kilander C, Strömberg R, Yngve A. Polyamine levels in breast milk are associated with mothers' dietary intake and are higher in preterm than full-term human milk and formulas. Journal of human nutrition and dietetics. 2014;27[5]:459-67.

[28] Darmon N, Drewnowski A. Does social class predict diet quality? The American journal of clinical nutrition. 2008;87[5]:1107-17. 